\title{
Oligosilane-nanofibers can be prepared through fabrication of permethyldecasilane within a helical superstructure of schizophyllan
}

\author{
Shuichi Haraguchi, Teruaki Hasegawa, Munenori Numata, Michiya Fujiki, Kazuya Uezu, \\ Sakurai Kazuo and Seiji Shinkai*
}

\section{Supporting Information}

Detailed description for the biphasic procedure

In this biphasic procedure, a hexane layer containing PMDS $(2.5 \mathrm{mg} / \mathrm{ml}, 20 \mu \mathrm{L})$ and aqueous sodium acetate $(0.33 \mathrm{M}$, $150 \mu \mathrm{l})$ were well homoginized by sonication and then, an aqueous $\mathrm{NaOH}(1.0 \mathrm{M}, 125 \mu \mathrm{L})$ containing s-SPG $(5.76$ $\mathrm{mg} / \mathrm{mL}$ ) were added. The resultant turbid mixture containing s-SPG and PMDS ([s-SPG]/[PMDS] $=1.2$ based on their repeating-units) was again well homoginized by sonication. Aqueous acetic acid (1.0 M, $125 \mu \mathrm{L})$ was then added to the resultant mixture to retrieve the original triple-stranded helical structure (t-SPG). After $12 \mathrm{~h}$ incubation, additional water $(1600 \mu \mathrm{L})$ and hexane $(1980 \mathrm{~mL})$ were added for easy separation of these two layers. The resultant diluted aqueous and hexane layers were separated from each other for their spectral measurements.

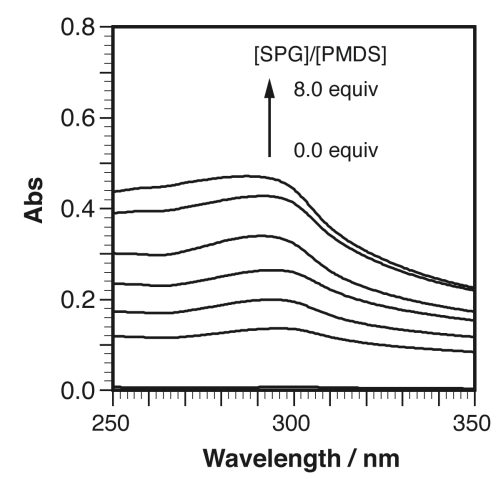

Fig. S1 (a) UV-vis spectra of the aqueous layer after the extraction procedure using different amounts of s-SPG: $d=5 \mathrm{~mm}, 25^{\circ} \mathrm{C}$, $[\mathrm{PMDS}]=0.431 \mathrm{mM}$ (an original concentration in the final hexane layers), [s-SPG] $=0.0 \sim 3.5 \mathrm{mM}(0.0 \sim 8.0$ equiv) (an original concentration in the final aqueous layers) based on their repeating units (four glucoside units for SPG and one dimethylsilane unit for PMDS).

Stoichiometric analysis of the composite

We also carried out detailed investigation on the SPG-PMDS stoichiometry in the SPG/PMDS composiotes. The SPG/PMDS composite was prepared through the biphasic procedure using excess amounts of s-SPG and the resultant aqueous layer was centrifuged (11000 rpm, $40 \mathrm{~min})$ to remove excess SPG. The pure SPG/PMDS composite was 
obtained through this treatment as white precipitate. SPG- and PMDS-fractions in the composite were quantified through two independent methods: that is, 1) the SPG-fraction was quantified according to the well-known phenolsulfric acid procedure that is frequently used to quantify carbohydrate-units and 2) the PMDS-fraction was quantified after dissociation of the composite in hexane and the resultant free (or liberated) PMDS can be easily quantified based on a intensity of the absorption band. These stoichiometric analysis revealed formation of 1:1 composites: that is, one PMDS molecule is included within the 1D cavity constructed by 10 SPG repeating units.

\section{Comparison between $\gamma \mathrm{CD} / \mathrm{PMDS}$ composite and SPG/PMDS composite}

Recently, Harada and co-workers reported an inclusion complex consinsing of $\gamma$-cyclodextrin $(\gamma \mathrm{CD})$ and PMDS. ${ }^{11}$ In this paper, they construct a possible model of the composite in which one PMDS molecule is threaded into a cavity of $\gamma \mathrm{CD}$ to form a neckless-like composite consisting of one PMDS molecule and several $\gamma \mathrm{CD}$ molecules. It is quite interesting to compare the structures between $\gamma \mathrm{CD} / \mathrm{PMDS}$ composite and SPG/PMDS composite in detail. UV-vis and CD spectral measurements revealed that $\gamma \mathrm{CD} / \mathrm{PMDS}$ composite prepared by Harada's procedure and our SPG/PMDS composite prepared by the biphasic procedure showed quite similar UV-vis and CD spectra, although the $\Delta \varepsilon / \varepsilon$-values of SPG/PMDS composite are $c a$. 10-fold smaller than those of the $\gamma \mathrm{CD} / \mathrm{PMDS}$ composite. These spectral similarity between these two composites again supports our model in which right- or left-handed helical PMDS molecules are included with in the 1D cavity of SPG.

(a)

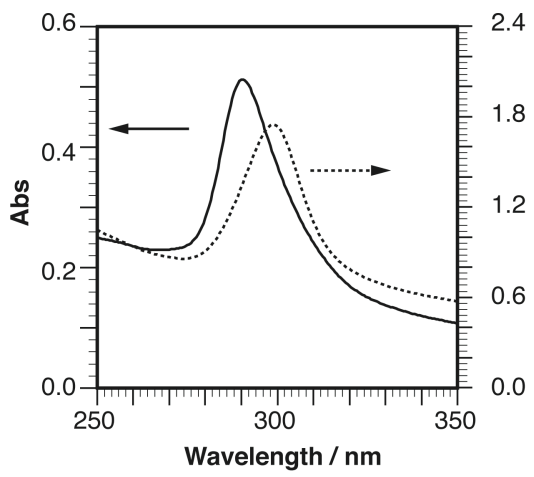

(b)

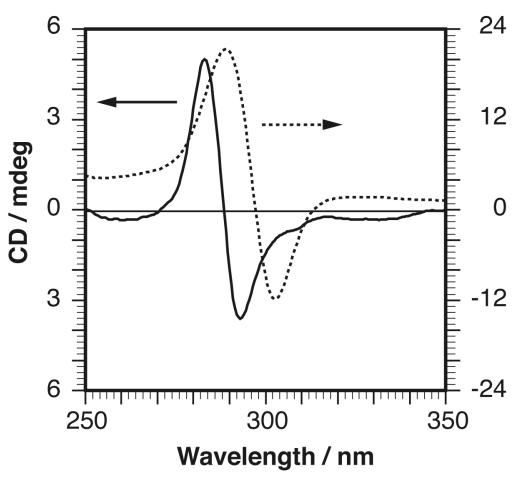

Fig. S2 (a) UV-vis spectra of (plane line) the SPG/PMDS composite and (dotted line) $\gamma$ CD/PMDS composite in aqueous solution and (b) the corresponding CD spectra: $d=5 \mathrm{~mm}, 25^{\circ} \mathrm{C}$, [PMDS] = unknown for the both composites, (for the SPG/PMDS composite) $[\mathrm{PMDS}]=0.4 \mathrm{mM}$ (an original concentration in the final hexane layers), $[\mathrm{s}-\mathrm{SPG}]=0.5 \mathrm{mM}$ (1.2 equiv) (an original concentration in the final aqueous layers) based on their repeating units (four glucoside units for SPG and one dimethylsilane unit for PMDS), $\left[\mathrm{CH}_{3} \mathrm{COONa}\right]=0.3 \mathrm{M}$, (for $\gamma \mathrm{CD} / \mathrm{PMDS}$ composite) $[\mathrm{CD}]=11.5 \mathrm{mM}$, $[\mathrm{PMDS}]=0.86 \mathrm{mM}$ (the original amount as a insoluble guest) 


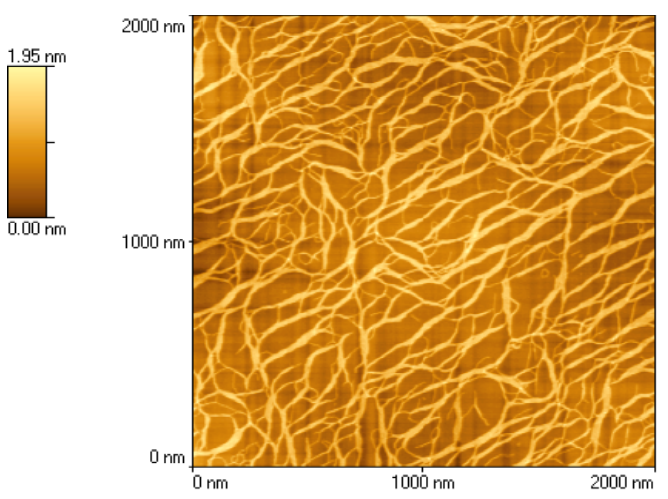

Fig. S3 AFM image of free SPG: on mica substrate.

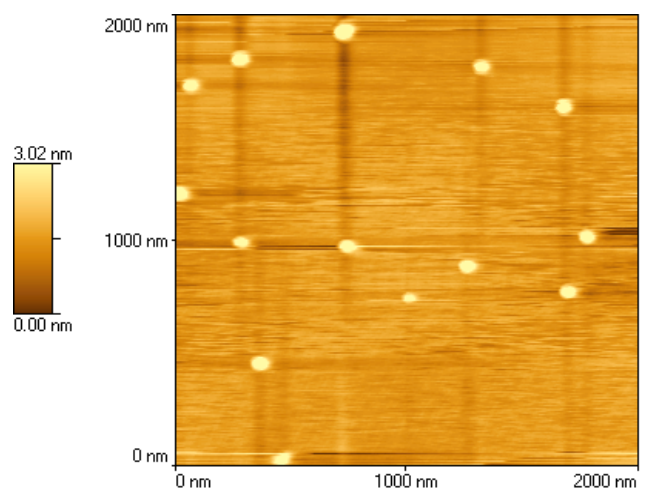

Fig. S4 AFM image of free PMDS: on mica substrate. 